\title{
New Research Perspectives on Thermostatted Kinetic Models
}

\author{
Carlo Bianca \\ Laboratory of Theoretical Physics of Condensed Matter, Sorbonne University, UPMC Univ Paris 06, \\ UMR 7600, 75252 Paris Cedex 05, France and CNRS, UMR 7600, LPTMC, Paris, France
}

Article history

Received: 22-01-2015

Revised: $18-05-2015$

Accepted: 27-05-2015

\begin{abstract}
This paper presents the new research directions that can be followed in the modeling of complex living systems by means of the tools of the thermostatted kinetic theory. The main aim is to invite the researchers that work in the field of applied mathematics to contribute to further developments of the theory with particular attention to applications in biology, crowds and swarm dynamics, economic and social systems.
\end{abstract}

Keywords: Kinetic Theory, Thermostat, Asymptotic Analysis, Nonlinearity, Complexity

\section{Introduction}

Recently the introduction of Gaussian thermostats in kinetic equations has gained much attention (Wennberg and Wondmagegne, 2004; 2006; Bagland et al., 2007; Degond and Wennberg, 2007). The Gaussian thermostat is a mathematical tool that allows to control the magnitude of energy of a system subjected to external force fields, see, among others, papers (Evans and Morriss, 1990; Morris and Dettmann, 1998; Jepps and Rondoni, 2006; Jepps et al., 2008; Jepps and Rondoni, 2010; Bianca, 2010; 2011) for the recent investigations and new developments.

A new thermostatted kinetic framework has been recently proposed in (Bianca and Lemarchand, 2014; Bianca et al., 2014b; Bianca, 2014) as general paradigm for the derivation of specific models in biological and chemical systems and the capability to model nonequilibrium stationary state has been shown in (Bianca, 2013). Specifically the new framework refers to systems constituted by a large number of entities, which are capable of interacting among themselves and with their outer environment. The method is based on the following steps:

Step 1: A preliminary phenomenological interpretation of the system and its complex characteristics (Bar-Yam, 2003)

Step 2: Subdivision of the overall system into functional subsystems. This splitting leads to the identification of functions that are expressed by the various components of the system and that have an important role in the overall dynamics. Functional subsystems are an aggregate of components, even characterized by different phenotype characterization, that collectively express the same functions

Step 3: Assessment of the scales that are necessary to represent the dynamics of each functional subsystem. Different scales may be necessary for different functional subsystems and, in some cases, even for the same subsystem. This aspect must be carefully taken into account. Moreover, the approach should consider the fact that the derivation of models at macroscopic scale is related to the lower scale, see (Bianca et al., 2014c; Bianca and Dogbe, 2015; 2014; Bianca and Lemarchand, 2015)

Step 4: Modeling of the dynamics of each functional subsystem. The derivation of models needs the mathematical description of the interactions involving entities of the same functional subsystems, but also interactions with the contiguous scales, lower and higher. Linear and nonlinear interactions can be considered (Bellomo, 2010). In general, these interactions have different outputs such as modification of the biological state, proliferative and/or destructive events, transition from one functional subsystem to the other (mutation), see (Schwartz, 2004; Nowak and Sigmund, 2004; Frank, 2007; Weinberg, 2007; Cooper, 2009)

Step 5: Validation of specific models. This is a very delicate issue considering that it must be based on empirical data related to experiments. A remarkable difficulty is that experiments in vitro refer to very special situations that only partially correspond to living reality. On the other hand, living systems show heterogeneous behavior that differentiates the response of each different individual 
Steps 1, 2, 3 and 5 have been already investigated, see (Bianca et al., 2014a). Therefore the new research perspectives proposed in this study will focus on the step 4 .

It is worth stressing that Journal of Mathematics and Statistics can be considered as a good platform for helping the community to give an answer to these open challenges.

\section{The Thermostatted Model and Research Perspectives}

The system is composed by a large number of interacting entities, called active particles, whose microscopic state, in addition to geometrical and mechanical variables, also includes an additional variable $u \in \mathbb{R}$, called activity, which represents the individual ability to express a specific strategy. Assume that the overall system is decomposed into $n \in \mathbb{N}$ functional distributions each of them subjected to a known external force field $F_{i}=F_{i}(u): D_{u} \rightarrow \mathbb{R}$. Let $f_{i}=f_{i}$ $(t, \mathrm{x}, \mathrm{v}, u)$, for $i \in\{1,2, \ldots, n\}$, be the distribution function of the particles of the $i$ th functional subsystem having at time $t$ the microscopic state $(\mathrm{x}, \mathrm{v}, u)$, where $(\mathrm{x}, \mathrm{v}) \in D_{\mathrm{x}} \times$ $D_{\mathrm{v}} \subset \mathbb{R}^{3} \times \mathbb{R}^{3}$ and $u \in D_{u} \subset \mathbb{R}$. The evolution equation for the distribution function $f_{i}$ is obtained by equating the time derivative of $f_{i}$ to the balance of the inlet and outlet flows into the elementary volume of the space of the microscopic states:

$$
\begin{aligned}
& \left(\partial_{t}+v \cdot \nabla_{x}\right) f_{i}+\partial_{u}\left(\left(F_{i}(u)-u \int_{\Omega} u F_{i}(u) \tilde{f} d x d v d u\right) f_{i}\right) \\
& =J_{i}[f]+N_{i}[f]+M_{i}[f]+v V_{i}\left[f_{i}\right]
\end{aligned}
$$

where, $\tilde{f}(t, x, v, u)=\sum_{i=1}^{n} f_{i}(t, x, v, u), \Omega=D_{x} \times D_{v} \times D_{u}$ and:

- $\quad \mathrm{v} \cdot \nabla_{\mathrm{x}} f_{i}$ is the transport operator;

- $D_{\mathrm{v}}$ is the velocity domain assumed bounded and spherically symmetric with respect to $(0,0,0)$;

- $\quad v$ is the turning rate of the velocity-jump $1 / v$ is the mean run time;

- $\quad J_{i}[f]=J_{i}[f](t, x, v, u)=G_{i}[f](t, x, v, u)-L_{i}[f](t, x, v, u)$ is the operator which models the conservative changing of the activity:

$$
\begin{aligned}
G_{i}[f]= & \sum_{j=l}^{n} \int_{D_{u} \times D_{u}} \eta_{i j}\left(u_{*}, u^{*}\right) A_{i j}\left(u_{*}, u^{*}, u\right) f_{i}\left(t, x, v, u_{*}\right) \times \\
& f_{i}\left(t, x, v, u^{*}\right) d u_{*} d u^{*} \\
L_{i}[f]= & f_{i}(t, x, v, u) \sum_{j=1}^{n} \int_{D_{u}} \eta_{i j}\left(u, u^{*}\right) f_{j}\left(t, x, v, u^{*}\right) d u^{*}
\end{aligned}
$$

where, $\eta_{i j}\left(u_{*}, u^{*}\right)$ is the interaction rate between the particle with microscopic state $\left(x, v, u_{*}\right)$ and the particle with microscopic state $\left(x, v, u^{*}\right)$ and $A_{i j}=A_{i j}\left(u_{*}, u^{*}, u\right): D_{u}$ $\times D_{u} \times D_{u} \rightarrow \mathbb{R}^{+}$is the probability density that a particle reaches the microscopic state $(x, v, u)$; in particular $\left\|A_{i j}\right\|_{L^{l}\left(D_{u}\right)}=1, \forall u_{*}, u^{*} \in D_{u}$.

- $\quad N_{i}[f]=N_{i}[f](t, x, v, u)$ is the operator which models proliferation or destruction of particles with microscopic state $(x, v, u)$ :

$$
\begin{aligned}
& N_{i}[f]=f_{i}(t, x, v, u) \sum_{j=1}^{n} \int_{D_{u}} \eta_{i j}\left(u, u^{*}\right) \mu_{i j}\left(u, u^{*}\right) \times \\
& f_{j}\left(t, x, v, u^{*}\right) d u^{*}
\end{aligned}
$$

where, $\mu_{i j}$ is the net proliferative/destructive rate.

- $\quad M_{i}[f]=M_{i}[f](t, x, v, u)$ is the operator which models proliferation or destruction of particles with microscopic state $(x, v, u)$ :

$$
\begin{aligned}
& M_{i}[f]=\sum_{h=1}^{n} \sum_{k=1}^{n} \int_{D_{u} \times D_{u}} \eta_{h k}\left(u_{*}, u^{*}\right) \varphi_{h k}^{i}\left(u_{*}, u^{*}\right) \\
& f_{h}\left(t, x, v, u_{*}\right) f_{k}\left(t, x, v, u^{*}\right) d u_{*} d u^{*}
\end{aligned}
$$

where, $\varphi_{h k}^{i}$ is the net mutation rate into the $i$ th functional subsystem, due to interactions that occur with rate $\eta_{h k}$ between the particles of the $h$ th functional subsystem and the $k$ th functional subsystem:

- $\quad V_{i}[f] \equiv V_{i}[f](t, x, v, u)$ is the operator which models the velocity-jump process:

$$
V_{i}\left[f_{i}\right]=\int_{D_{v}}\left[T_{i}\left(v^{*}, v\right) f_{i}\left(y, x, v^{*}, u\right)-T_{i}\left(v, v^{*}\right) f_{i}(t, x, v, u)\right] d v^{*}
$$

where $T_{i}\left(v^{*}, v\right)$ is the turning kernel which gives the probability that the velocity $\mathrm{v} \in D_{v}$ jumps into the velocity $\mathrm{v} \in D_{v}$ (if a jump occurs).

- The thermostatted operator reads:

$$
T_{F i}[f]=\partial_{u}\left(\left(F_{i}(u)-u \int_{\Omega} u F_{i}(u) \tilde{f}(t, x, v, u) d x d v d u\right) f_{i}(t, x, v, u)\right)
$$

which is a damping operator.

The local $p$ th-order moment of the $i$ th functional subsystem and with respect to the activity variable is defined as follows: 


$$
E_{p}\left[f_{i}\right](t, x)=\int_{D_{u} \times D_{v}} u^{p} f_{i}(t, x, v, u) d u d v
$$

The main interest is focused on the first-order and second-order moments, namely the mean activation and activation energy that correspond in mechanics to linear momentum and kinetic energy.

New research directions refer to further developments of the above framework in particular for what concern the introduction of weighted (multiple) interactions.

\section{How to Introduce Nonlinear Interactions}

In order to consider the role of nonlinear interactions, an important concept that is useful to the modeling of complex living systems is the definition of a distance $d_{i j}$ between the active particles of the $i$ th and the $j$ th functional subsystems. This step depends on the specific characteristics of the system under consideration. In particular the modeling of the encounter rate $\eta_{i j}$ can be achieved in a fashion such that increasing values of the distance $d_{i j}$ corresponds to decreasing values of the encounter rate $\eta_{i j}$. A high nonlinearity is also introduced when the probability density $A_{i j}$ is conditioned by the distribution functions of the interacting functional subsystems and its momenta, e.g., $A_{i j}$ $\left(u_{*}, u^{*}, u \mid \mathbb{E}_{p}\left[f_{i}\right], \mathbb{E}_{p}\left[f_{j}\right]\right)$.

A phenomenon which has to be taken into account is that the interaction domain of the particle with state $u_{*}$ is not the whole domain $D_{u}$ but a subset $\Omega_{u_{*}} \subseteq D_{u}$, which contains the particles with activity $u^{*} \in \Omega_{u_{*}}$ that are able to interact with the particles with activity $u_{*}$. Thus interactions only occur if the distance, in the space of microscopic states of the interacting particles, is sufficiently small.

Bearing all above in mind, a positive function $\omega\left(u_{*}, u^{*}\right)$, normalized with respect to integration over $u^{*}$, is introduced to take into account such dynamics. This function, which weights the interactions among the active particles, is assumed to have a compact support in the domain of influence $\Omega_{u^{*}} \subseteq D_{u}$ of the interactions. Moreover:

$$
\int_{D_{u}} \omega\left(u_{*}, u^{*}\right) d u^{*}=\int_{\Omega_{u^{*}}} \omega\left(u_{*}, u^{*}\right) d u^{*}=1
$$

Accordingly we define the local pth order weighted moment as follows:

$$
\begin{aligned}
& E_{p, w}\left[f_{i}\right]\left(t, x, u_{*}\right)=\int_{D_{u}}\left(u^{*}\right)^{p} \omega\left(u_{*}, u^{*}\right) f_{i}\left(t, x, v, u^{*}\right) d u^{*} d v \\
& \int_{\Omega_{*}}\left(u^{*}\right)^{p} \omega\left(u_{*}, u^{*}\right) f_{i}\left(t, x, v, u^{*}\right) d u^{*} d v
\end{aligned}
$$

\section{How to Introduce Multiple Interactions}

The active particle with microscopic state $\left(x, v_{*}, u_{*}\right)$ interacts with the particles having microscopic state $\left(x^{*}, v^{*}\right.$, $\left.u^{*}\right)$ located in its interaction domain $\Lambda_{*}$. In this case the interaction rate depends on the local density, namely $\eta\left[E_{0}\right.$ $\left.\left[f_{i}\right], E_{0}\left[f_{j}\right]\right]\left(t, x^{*}\right)$. The distance and topological distribution of the intensity of the interactions is weighted by a function $\sigma\left(x, x^{*}\right)$ such that:

$$
\int_{\Lambda_{*}} \sigma\left(x, x^{*}\right) d x^{*}=1
$$

Accordingly, the gain term $G_{i}$ now reads:

$$
\begin{aligned}
& G_{i}[f]=\sum_{j=1}^{n} \int_{\Omega_{u_{*} \times \Lambda_{*}}} \int_{D_{u} \times D_{u}} \omega\left(u_{*}, u^{*}\right) \sigma\left(x, x^{*}\right) \\
& \eta\left[f_{i}, f_{i}, E_{0}\left[f_{i}\right], E_{0}\left[f_{j}\right]\right]\left(t, x^{*}\right) \times \\
& A_{i j}\left(u_{*}, u^{*}, u \mid E_{p}\left[f_{i}\right]\right), E_{p}\left[f_{j}\right]\left(t, x, v_{*}, u_{*}\right) \times \\
& f_{j}\left(t, x^{*}, v^{*}, u^{*}\right) d x^{*} d v_{*}, d v^{*} d u_{*} d u^{*}
\end{aligned}
$$

From the theoretical point of view there are many interesting investigations that have to be pursued in this new thermostatted framework, for example:

- The problem of the existence and uniqueness of weak or strong solutions of the related Cauchy problem; this is a hard issue considering the problem of integration along the characteristic and the introduction of high nonlinearities;

- The proof that the thermostatted framework preserves the energy of the system or in general some momentum of the solution;

- The mathematics analysis related to the asymptotic behavior of the solution, including blow-up;

- The proof of the existence of nonequilibrium stationary states and the related convergence;

- The derivation of macroscopic equations for momenta of the solution by employing parabolic and hyperbolic scalings.

From the applications point of view there are other systems that can be modeled by employing, with suitable modifications, this new thermostatted framework, for example:

- Vehicular traffic and crowd dynamics under panic conditions;

- Aggregation and/or fragmentation phenomena which are typical of a wide variety of physical situations where clusters (or particles, or droplets) merge by coalescence to form larger ones;

- Social and economic systems with particular attention to political conflicts. 
It is worth stressing that the developed methods and tools will hopefully suggest new directions to the modelling of complex living systems.

\section{Acknowledgement}

The author thanks the partial support by L'Agence Nationale de la Recherche (ANR T-KiNeT Project).

\section{Ethics}

This article is original and contains unpublished material. The corresponding author confirms that all of the other authors have read and approved the manuscript and no ethical issues involved.

\section{References}

Bagland, V., B. Wennberg and Y. Wondmagegne, 2007. Stationary states for the noncutoff Kac equation with a Gaussian thermostat. Nonlinearity, 20: 583-604. DOI: 10.1088/0951-7715/20/3/003

Bar-Yam, Y., 2003. Dynamics of Complex Systems. Studies in Nonlinearity. 1st Edn., Westview Press, ISBN-10: 0813341213, pp: 864.

Bellomo, N., C. Bianca and M.S. Mongiovì, 2010. On the modeling of nonlinear interactions in large complex systems. Applied Math. Lett., 23: 1372-1377.

DOI: 10.1016/j.aml.2010.07.001

Bianca, C. and A. Lemarchand, 2014. A kinetic framework for modeling nonequilibrium biological and chemical systems. Nonlinear Stud., 21: 367-374.

Bianca, C. and A. Lemarchand, 2015. Density evolution by the low-field limit of kinetic frameworks with thermostat and mutations. Communi. Nonlinear Sci. Numerical Simulation, 20: 14-23. DOI: $10.1016 /$ j.cnsns.2014.05.009

Bianca, C. and C. Dogbe, 2014. Kinetic models coupled with Gaussian thermostats: Macroscopic frameworks. Nonlinearity, 27: 2771-2803. DOI:10.1088/0951-7715/27/12/2771

Bianca, C. and C. Dogbe, 2015. Mean-field limit of a microscopic individual-based model describing collective motions. J. Nonlinear Math. Phys., 22: 117-143. DOI: 10.1080/14029251.2015.996444

Bianca, C., 2010. On the mathematical transport theory in microporous media: The billiard approach. Nonlinear Analysis: Hybrid Syst., 4: 699-735. DOI: 10.1016/j.nahs.2010.04.007

Bianca, C., 2011. Weyl-flow and the conformally symplectic structure of thermostatted billiards: The problem of the hyperbolicity. Nonlinear Analysis: Hybrid Syst., 5: 32-51.

DOI: $10.1016 /$ j.nahs.2010.08.004
Bianca, C., 2013. Existence of stationary solutions in kinetic models with Gaussian thermostats. Math. Methods Applied Sci., 36: 1768-1775. DOI: $10.1002 / \mathrm{mma} .2722$

Bianca, C., 2014. How do mutative events modify moments evolution in thermostatted kinetic models? Communications in Nonlinear Sci. Numerical Simulation, 19: 2155-2159. DOI:10.1016/j.cnsns.2013.12.009

Bianca, C., C. Dogbe and L. Guerrini, 2014a. A thermostatted kinetic framework with particle refuge for the modeling of tumors hiding. Applied Math. Inform. Sci., 8: 469-473. DOI: 10.12785/amis/080203

Bianca, C., M. Ferrara and L. Guerrini, 2014b. Highorder moments conservation in thermostatted kinetic models. J. Global Optimization, 58: 389-404. DOI: $10.1007 / \mathrm{s} 10898-013-0045-2$

Bianca, C., M. Ferrara and L. Guerrini, 2014c. The asymptotic limit of an integro-differential equation modelling complex systems. Izvestiya: Mathe, 78: 1105-1119. DOI: 10.1070/IM2014v078n06ABEH002722

Cooper, E.L., 2009. Evolution of immune systems from self/not self to danger to artificial immune systems. Phys. Life Rev., 7: 55-78. DOI:10.1016/j.plrev.2009.12.001

Degond, P. and B. Wennberg, 2007. Mass and energy balance laws derived from high-field limits of thermostatted Boltzmann equations. Commun. Math. Sci. 5: 355-382. DOI: 10.4310/CMS.2007.v5.n2.a7

Evans, D.J. and G.P. Morriss, 1990. Statistical Mechanics of Nonequilibrium Liquids. 1st Edn., Academic Presss, London, ISBN-10: 0122440900, pp: 302.

Frank, S.A., 2007. Dynamics of Cancer: Inheritance and Evolution. 1st Edn, Princeton University Press, Princeton, ISBN-10: 0691133662, pp: 378.

Jepps, O. and L. Rondoni, 2006. Thermodynamics and complexity of simple transport phenomena. J. Phys., A: Math Gen., 39: 1311-1338. DOI: $10.1088 / 0305-4470 / 39 / 6 / 007$

Jepps, O., C. Bianca and L. Rondoni, 2008. Onset of diffusive behavior in confined transport systems. Chaos, 18: 013127. DOI: 10.1063/1.2888759

Jepps, O.G. and L. Rondoni, 2010. Deterministic thermostats, theories of nonequilibrium systems and parallels with the ergodic condition. J. Phys. A: Math. Theor., 43: 133001.

DOI: $10.1088 / 1751-8113 / 43 / 13 / 133001$

Morris, G.P. and C.P. Dettmann, 1998. Thermostats: Analysis and application. Chaos, 8: 321-336. DOI: $10.1063 / 1.166314$ 
Nowak, M.A. and K. Sigmund, 2004. Evolutionary dynamics of biological games. Science, 303: 793-799. DOI: 10.1126/science.1093411

Schwartz, L., 2004. Cancer Between Glycolysis and Physical Constraint. 1st Edn., Springer-Verlag Berlin Heidelberg,

ISBN-10: 978-3-642-18543-4, pp: 150.

Weinberg, R.A. 2007. The biology of cancer. Taylor and Francis, New York.
Wennberg, B. and Y. Wondmagegne, 2004. Stationary states for the Kac equation with a Gaussian thermostat. Nonlinearity, 17: 633-648. DOI: $10.1088 / 0951-7715 / 17 / 2 / 016$

Wennberg, B. and Y. Wondmagegne, 2006. The Kac equation with a thermostatted force field. J. Statistical Phys., 124: 859-880.

DOI: $10.1007 / \mathrm{s} 10955-005-9020-8$ 